\title{
Real-time content-aware texturing for deformable surfaces
}

\author{
Charalampos Koniaris \\ University of Bath \\ dpc@cs.bath.ac.uk \\ Kenny Mitchell \\ Disney Research \\ kmitchell@disneyresearch.com
}

\author{
Darren Cosker \\ University of Bath \\ dpc@cs.bath.ac.uk
}

\author{
Xiaosong Yang \\ University of Bournemouth \\ xyang@bournemouth.ac.uk \\ lain Matthews \\ Disney Research \\ iainm@disneyresearch.com
}

\begin{abstract}
Animation of models often introduces distortions to their parameterisation, as the parameterisation has been optimised for a single frame. When mapping textures or displacements on a deforming surface with such a constant parameterisation, distortions manifest visually as texture-mapped features appearing uniformly elastic, and such behaviour is not always desired. In this paper we introduce a real-time technique that reduces such parameterisation distortions in areas specified in a provided distortion control (rigidity) map. The parameter space is warped in an axis-aligned way to minimise a non-linear distortion metric using a hybrid CPU-GPU solver. We also extend the technique to compute arbitrary warps for handling more complex use cases. The result is real-time dynamic content-aware texturing that reduces distortions in a controlled way. The technique can be applied to reduce distortions in a variety of scenarios where highly detailed rigid features are represented on a map, abstracted from the underlying low-complexity deforming geometry they are mapped on. Such scenarios include reusing a low geometric complexity animated sequence with a multitude of detail maps, dynamic procedurally defined features mapped on deformable geometry and animation authoring previews on texturemapped models.
\end{abstract}

\section{INTRODUCTION}

Texture mapping is the process of mapping detail to a surface using a parameterisation of the surface, the most common case being a 2D parameterisation of a 3D surface [7]. Some surface representations have natural parameterisations (e.g. NURBS), while others, such as polygonal meshes, require non-trivial methods to obtain such parameterisations. In the latter group, parameterisations are represented in the same way as vertices are: as piecewise-linear approximations to continuous functions. Naturally, dense discretisations of these functions provide higher-quality approximations. A metric for the quality of a parameterisation is the distortion introduced by the mapping [14]. Another source of distortion is the error introduced by the piecewise-linear approximation. Both of these sources of distortion depend on the parameterisation algorithm used, as well as the 3D surface discretisation.
An additional common source of distortion in the parameterisation is caused by animating the mesh (figure 2). As the mesh undergoes a non-Euclidean transform, the parameterisation ceases to be optimal. This manifests visually as mapped features on the deforming primitives appearing elastic. That can be desired in some cases (skin, rubber, etc) but not all: if such mapped features need to appear rigid, this elastic behaviour causes suspension of disbelief. To reduce this type of distortion, either the parameterisation needs to be regenerated, or the mesh needs to be manually edited, as explained in section 2 .

We introduce a novel method to reduce distortions caused by the deformation of an already parameterised surface in real-time, as the surface deforms. The distortions are reduced over a user-specified rectangle in texture space ensuring optimisation is locally contained in areas of interest. The deformation of the surface does not need to be known a priori. The distortion minimisation algorithm is guided by a user-supplied distortion control map of the specified region of interest (ROI). The distortion control map and region are supplied as a single preprocessing step. Such user interaction is simple to do and provides more flexibility in deformations compared to adding more vertices. For a given animation frame, we use non-linear optimisation to calculate a piecewise-linear warp that, when applied to the ROI's parameterisation, reduces distortions introduced by the current frame's surface deformation. We provide two variants of the algorithm that trade off between performance and quality: an axis-aligned warp, in the sense that it deforms a rectilinear grid mapped on the ROI to another rectilinear grid by calculating new horizontal and vertical grid line offsets, and a non-axis-aligned warp, where the grid is arbitrarily deformed to another grid with the same connectivity.

Our method abstracts the distortion correction from the geometric representation of the surface, providing flexibility over mesh-based approaches as it does not require editing of the surface. The use of a dynamic spatially varying distortion control provides additional flexibility over the distribution of distortions after the reparameterisation, allowing preservation of particular features on a map at any given time. The user interaction is kept low, as it consists of selecting the ROIs and providing distortion control maps as a preprocess. The storage cost of the reparameterisation is very low, as it only requires a sparse set of lines (axis-aligned) or points (nonaxis-aligned). The runtime cost depends on the dimensions of the grid, and can be very low if the results have been precalculated.

\section{RELATED WORK}

Parameterisation distortions are typically minimised using mesh parameterisation algorithms $[8,20,5]$. The majority of such algo- 

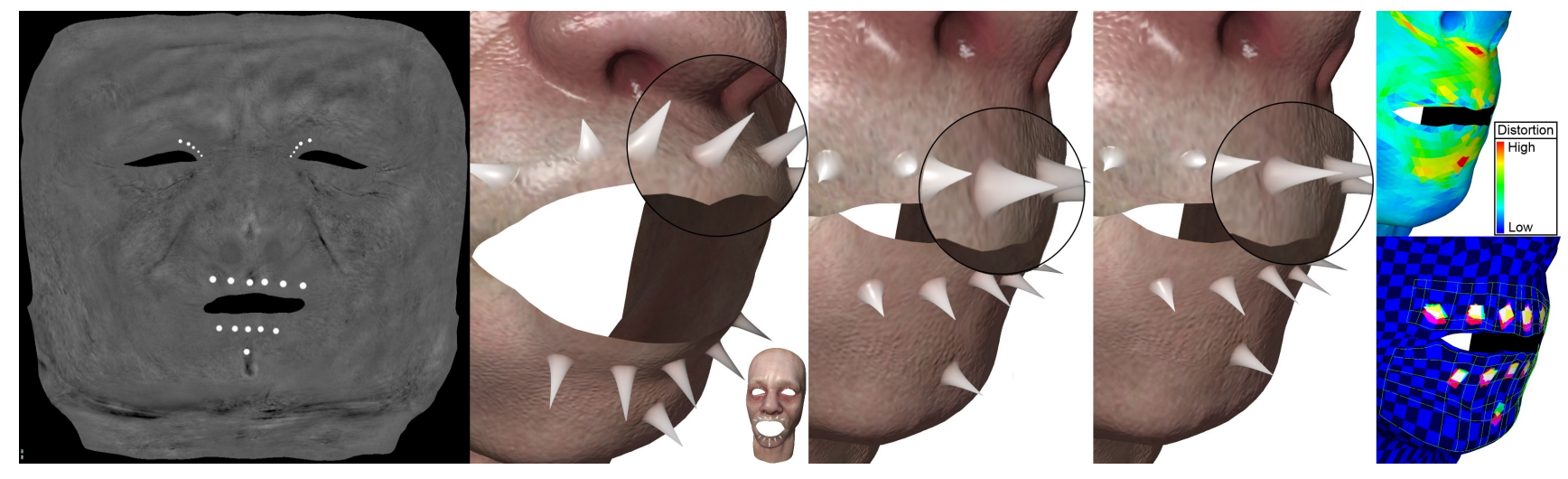

Figure 1: Spikes on an animated face. The spikes are modelled as a displacement map (left) mapped on the rest pose (middle-left). Deforming the face introduces distortion to the parameterisation (top-right), causing the skin and spikes to stretch (middle). Our algorithm automatically corrects the parameterisation in real-time, so that only the spikes remain rigid (middle-right). The parameterisation is corrected in regions of interest given distortion control maps, which specify features that need to remain undistorted, and overlaying and warping rectilinear grids (bottom-right).

rithms minimise distortion metrics over the whole domain, without taking into account the nature of the data that are mapped using the parameterisation.

Sander et al. [18] minimise a signal-stretch metric that allows reduction of distortions of any vector-valued function (the signal) defined over the domain. The metric is non-linear and the process requires a few minutes per model. While the technique is contentaware, it does not take into account temporal coherence of the parameterisation.

Sheffer and De Sturler [19] overlay a 2D uniform Cartesian grid on the texture parameterisation domain (as a 2D triangle mesh) and warp the grid so that the warped parameterisation minimises edge length distortions. While this technique is similar in ours in the fact that it uses an overlayed grid to warp the parameterisation, it is not content-aware, as it does not take into account either rigidity or sliding of features. Also, our technique is about three orders of magnitude faster, allowing the parameterisation warping to run in real-time.

Ptex [4], by Burley and Lacewell, eliminates the need for explicit parameterisation by using the natural parameterisation of subdivision surface quad faces and providing anisotropic filtering between faces. While this eliminates many of the explicit parameterisation issues, such as distortions and seams, animated meshes still pose a problem, as the individual quads still deform and distortions are reintroduced.

If the parameterisation needs to remain constant, mesh deformation techniques can be employed to calculate a further constrained deformation, so that parameterisation distortions remain low. Such techniques $[3,21,22]$ focus on editing complex meshes in a physically plausible and aesthetically pleasing way, while preserving geometric details. Barycentric coordinates are also used for mesh deformation, by deforming complex meshes using simple control cages $[11,10,13,2,9]$. While these techniques are generally efficient in calculating a new plausible mesh pose given a few control transformations, they do not address the case where the deformed coarse mesh is entirely known, but the deformed fine-scale details are not.
Popa et al. [17] approach content-aware deformation by introducing local bending and shearing stiffnesses as factors in how a mesh deforms. Given such material information, and transformations for a number of anchor triangles, they calculate the deformation of the mesh as a weighted sum or blend of the anchor transformations. The material information is user- or data-driven, providing additional control on how parts of the mesh deform when editing it. As the anchor transformations are required to be a combination of rotations and uniform scales, the space of supported deformations is restricted.

Kraevoy et al. [12] focus on protecting vulnerable parts of a complex model under global non-uniform scaling. They define a vulnerability map on a volumetric grid that encloses the object, and transform the grid while respecting this map. While they estimate vulnerability based on slippage and normal curvature, the map can be user-driven. The technique focuses only on a very special deformation case (non-uniform scaling transform), so it's not applicable to more complex deformations.

Yang et al. [23] simulate skin sliding by remeshing the surface based on resampling of the its parameter space. They use the Force Density Method (FDM) to construct embeddings of original and deformed patches into their parameter domains. As the technique deforms the actual geometry and force densities are specified on edges, so the deformed patch needs to be highly tessellated and the result is dependent on the triangulation, which reduces the flexibility and applicability of the method.

In production, in order to reduce texture space distortions in sensitive regions of an animated mesh, artists need to manually add vertices, tightly bounding the rigid area and making sure that it does not distort under deformation. For example, for rigged models, the regions around joints are the most prone to distortions, so additional vertices need to be placed there. When the deformation is known, additional vertices need to be placed appropriately so that deformation is spread to areas that do not contain any salient rigid features. The problem remains when the deformation is unknown or varying so much that adding vertices becomes impractical. Procedurally generated detail, static or animated, provides an even greater challenge as the location of the additional vertices cannot be easily determined. 


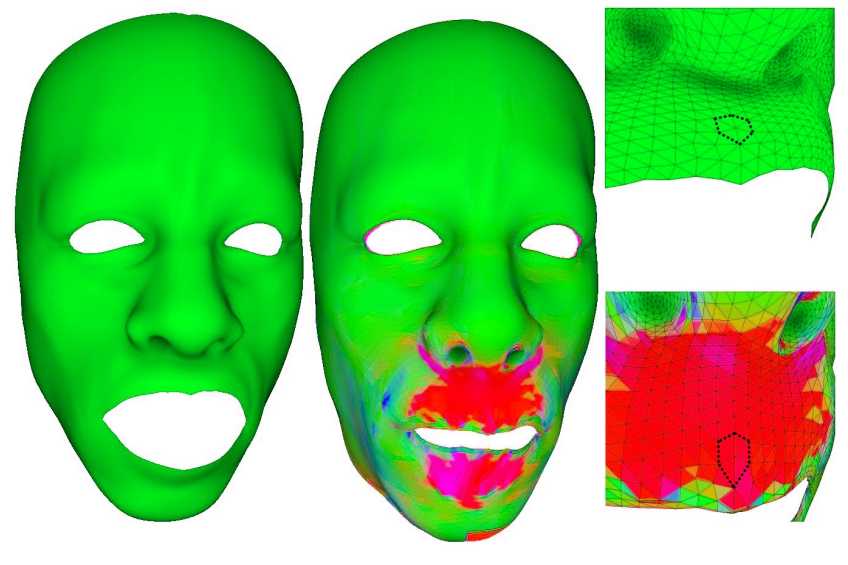

Figure 2: Texture space distortion in animation. The rest pose is shown on the left. Another animation frame is shown in the middle, where deformation of texture space relatively to the chosen rest pose is marked with blue ( $u$ axis), red ( $v$ axis) and purple (both axes). On the right the zoomed-in area under the nose for the original (top-right) and the deformed (bottom-right) frames are shown, along with a highlighted vertex neighbourhood. Note that such distortion can be desired if the mapped material needs to behave in an elastic way (skin, rubber, etc).

\subsection{Contributions}

We present an algorithm that, guided by a distortion control map, dynamically modifies the parameterisation of a deformable mesh so it reduces distortions caused by the deformation only in areas specified in the map. The distortion control map as well as the deformed frame of the mesh do not need to be known a priori, as our algorithm works with fully dynamic data but is optimised for known, constant distortion control maps. We decribe two versions of the algorithm: both warp a rectilinear grid overlayed on the parameterisation, one in an axis-aligned way (real-time, low storage) and one using arbitrary warping (offline/interactive, better quality). To our knowledge, we are the first to present such a real-time, automated reparameterisation method guided by distortion control data.

\section{OVERVIEW}

This article proposes a reparameterisation method applicable to all parameterised, deformable surfaces, which takes into account salient, rigid features of a given static or animated detail map. We borrow ideas from image retargetting $[6,16]$ as we calculate a content-aware warp of the $2 \mathrm{D}$ parameterisation domain to minimize a specified distortion energy functional. The reparameterisation process (figure 3 ) can be briefly described as a series of steps as follows:

1. User input. A rectangular region is initially selected from the $2 \mathrm{D}$ parameterisation domain; this is the region that the algorithm will process. We map this region to the unit square. The rigidities associated with the ROI can also be provided at this stage.

2. Preprocessing. We partition the ROI domain to a rectilinear grid. If rigidities are provided here, the grid is created by clustering similar distortion control weights to grid cells (section 4).
3. Per-frame processing. The rigidities and the grid line coordinates are used as an input to the optimiser, which minimises texture-space deformation energy by adjusting the line coordinates while keeping the domain boundary constant (section 5).

4. Per-frame rendering. After the deformed grid is calculated, we can render the surface using the adjusted parameterisation (section 6).

\subsection{Notation}

The ROI is expressed in 3D object space as $O(s, t)$ (rest pose) and $D(s, t)$ (deformed pose), where $(s, t)$ are parametric coordinates on the unit square. The corresponding region in the $2 \mathrm{D}$ parameterisation is expressed as $T(s, t)$. The distortion control map is expressed as $R(s, t)$, containing values between 0 (non-rigid) and 1 (rigid). Partial derivatives for any function $X(s, t)$ are written as $X_{s}^{\prime}(s, t)$ and $X_{t}^{\prime}(s, t)$. The dimensions of the distortion control map that we use are $W \times H$ and the dimensions of the rectilinear grid are $M \times N$ (horizontal and vertical lines). Unless noted otherwise explicitly, we will be using zero-based indexing. The $2 \mathrm{D}$ unit domain axes are specified as $\hat{\mathbf{s}}$ (horizontal) and $\hat{\mathbf{t}}$ (vertical). The 1D solutions for each axis are represented as $\mathbf{S}$ and $\mathbf{t}$, and have lengths of $M$ and $N$ respectively, while for the $2 \mathrm{D}$ variant, both solutions have dimensions of $M \times N$ each. As the formulas for the calculation of both axes are in many cases similar, we will present formulas for a single axis and note the similarity.

\section{RECTILINEAR GRID PARTITIONING}

As we remap the parameter space of the ROI, the shape of the region can be any shape that can be bijectively mapped to a rectangle. In our examples, we use scaled and rotated rectangles for their simplicity of converting between texture coordinates and $(s, t)$ parametric coordinates (figure 4).

As the grid optimisation performance greatly depends on the grid resolution, we need to use as few lines as possible. If a distortion control map is provided at this stage, grid lines can be chosen so that the resulting cells enclose as-similar-as-possible distortion control weights and rigid areas are enclosed in cells as tightly as possible. For relatively simple cases the grid line offsets can be automatically calculated with algorithm 1 , otherwise it can be provided by a user.

This can be performed once as a preprocessing step, if the mapped detail (and thus the distortion control map) remains constant throughout deformation.

\section{GRID OPTIMISATION}

In this section we show how we formulate the distortion energy and constraints to a non-linear problem.

\subsection{Energy formulation}

The distortion metric that we use is how close does the deformed remapped surface match the original in terms of stretch, along the $\hat{\mathbf{s}}$ and $\hat{\mathbf{t}}$ directions. We define the total per-axis distortion energy as the sum of the individual per-cell, per-axis distortion energies. The horizontal energy is: 


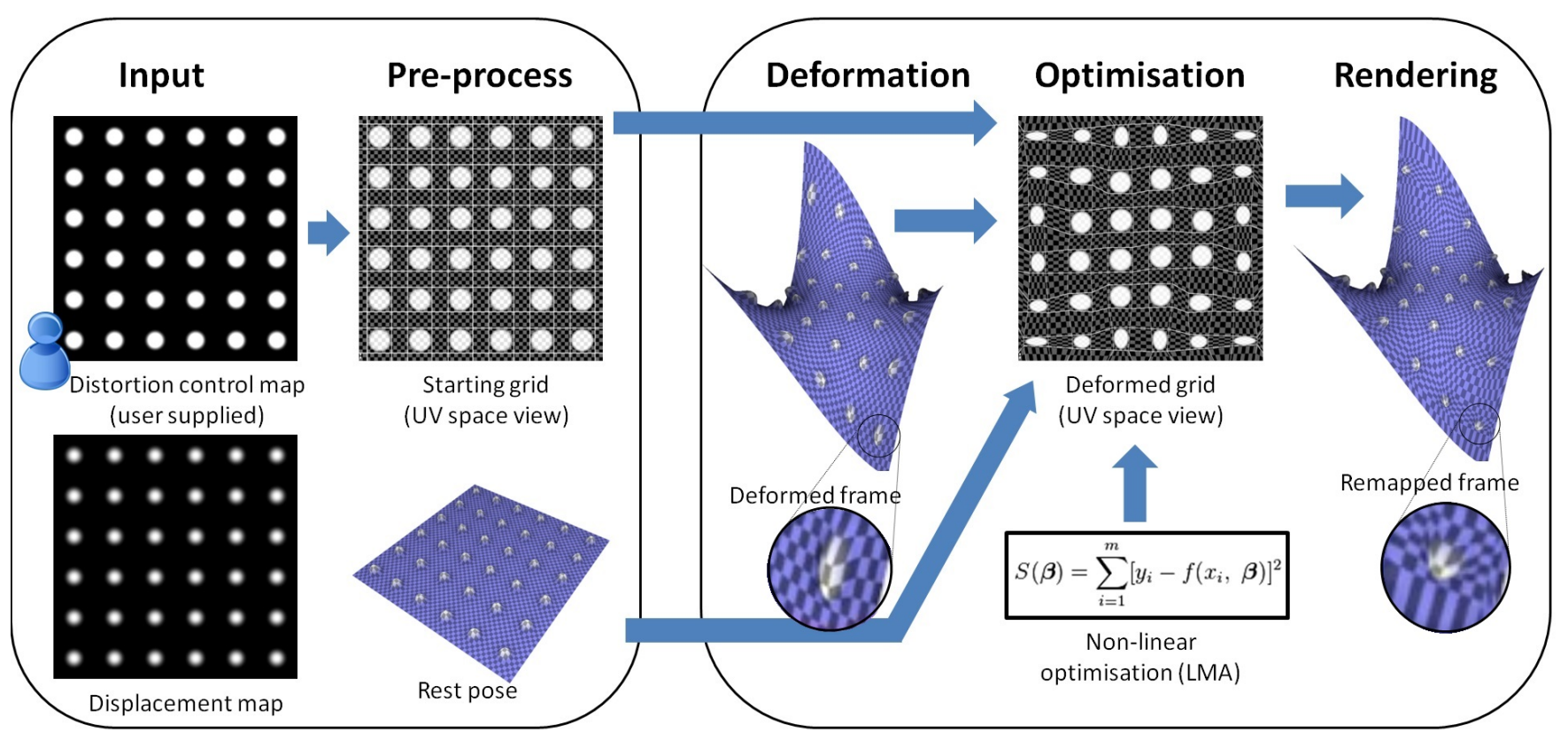

Figure 3: The process flow. The starting grid is generated as a pre-process, given the distortion control map. The distortion control map is specified so that the bumps on the plane are preserved. When deforming, the original surface, deformed surface, distortion control map and starting grid are all used by the non-linear optimiser to calculate a new grid that minimizes a weighted distortion energy functional. The starting and warped grids are then used to warp the parameterisation function, which is used to sample the mapped surface detail when rendering.

Data: $R, W, H, e$

Result: s,t

// Maximum per-column distortion control weight

for each column $j=0 \rightarrow(W-1)$ do

$r_{j}=\max _{i \in[0, H-1]} R(i, j)$;

end

// Calculate $s$ lines

$s_{0} \leftarrow 0$

$i d x \leftarrow 1$;

for each column $j=0 \rightarrow(W-1)$ do

if $\left\|r_{j}-r_{j+1}\right\|>e$ then

if $r_{j}>r_{j+1}$ then

$s_{i d x} \leftarrow \frac{j}{W-1}$;

else

$\mid s_{i d x} \leftarrow \frac{j+1}{W-1}$

end

$i d x \leftarrow i d x+1$

end

end

$s_{i d x} \leftarrow 1$

// ...Similarly for $t$ lines

Algorithm 1: Generating the non-uniform grid from a distortion control map $R$ of size $W \times H$ with values in $[0,1]$ given a threshold $e$.

$$
E_{s}=\sum_{i=0}^{N-1} \sum_{j=0}^{M-1} E_{s_{i j}}
$$

where the per-cell energy is defined as:

$$
E_{S_{i j}}=\int_{t=t_{i}}^{t_{i+1}} \int_{s=s_{i}}^{s_{i+1}} R(s, t) F_{s}(s, t) d s d t
$$

where $F_{S}(s, t)$ calculates stretch at a point $(s, t)$ as the squared weighted difference of the lengths of the original and deformed/remapped geometric partial derivative along the $\hat{\mathbf{s}}$ direction at that point:

$$
F_{s}(s, t)=\left(\left|f^{\prime}(s)\right|\left\|D_{s}^{\prime}(f(s), g(t))\right\|_{2}-\left\|O_{s}^{\prime}(s, t)\right\|_{2}\right)^{2}
$$

where $f, g$ are the linear functions that remap $s$ and $t$ for the given cell. Similarly for the vertical energy:

$$
F_{t}(s, t)=\left(\left|g^{\prime}(t)\right|\left\|D_{t}^{\prime}(f(s), g(t))\right\|_{2}-\left\|O_{t}^{\prime}(s, t)\right\|_{2}\right)^{2}
$$

It can be seen that movement of vertical lines does not result in any horizontal energy change and vice versa. Derivation of the energy can be found in appendix A.

\subsection{Constraints}



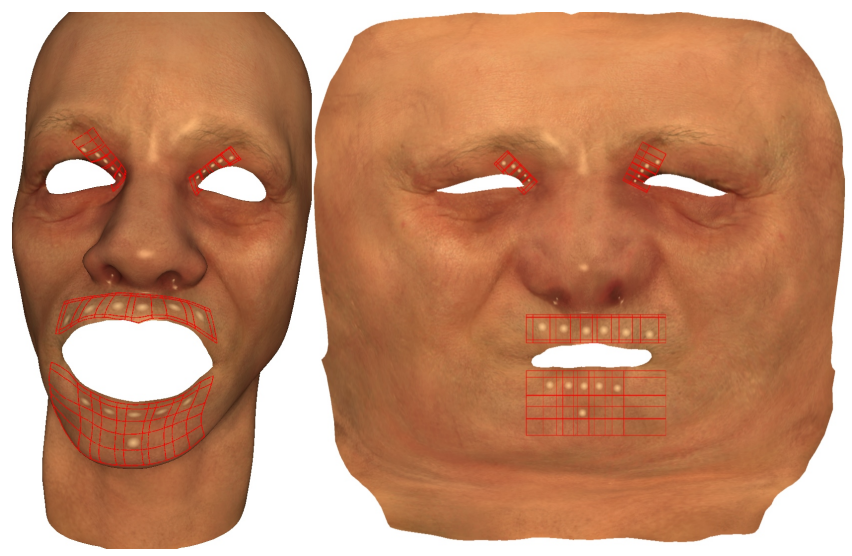

Figure 4: Four ROIs and starting grids in object space (left) and texture space (right) enclosing rigid points (shown in white) using the mesh of figure 1 .

We want to calculate axis-aligned grid lines that minimise the above distortion energy and satisfy the following requirements: a) no line foldovers b) smooth line changes from frame to frame c) prefer local minima and d) allow "seamless" solutions for looping animations. The constraints can be formed as boundary constraints, as they are compatible with local solutions and allow for faster optimisation. Below, we show constraints for the $\hat{\mathbf{s}}$ axis only, and refer to previous (known) and current (unknown) solutions as $\mathbf{s}^{k}$ and $\mathbf{s}^{k+1}$ respectively.

Foldover constraints prevent discontinuities in the remapping and are easily enforced by bounding a line between the midpoints of the segments between the line and its adjacent neighbours:

$$
\frac{s_{i-1}^{k}+s_{i}^{k}}{2}<s_{i}^{k+1}<\frac{s_{i+1}^{k}+s_{i}^{k}}{2}
$$

Smooth line changes can simply be enforced by restricting the movement of a line in a solution to a maximum offset $o$ :

$$
s_{i}^{k}-o<s_{i}^{k+1}<s_{i}^{k}+o
$$

The above constraints result in local solutions, so the local minima requirement is satisfied. For a looping animation consisting of $K$ frames we add the following constraint for the $j$-th frame:

$$
d=\frac{K-|2(j+1)-K+1|-1}{2}
$$

where $s^{0}$ is the solution for the first (or last) frame. This constraint effectively shifts the bounds so that the first and last solutions are matching, and solutions in between vary smoothly.

As the intersection of all these ranges might be $\emptyset$, we need to define a behaviour that gives precedence to a constraint over another. Given constraints of descending priority $C_{h}, C_{l}$, a merged constraint can be calculated as follows:

$$
F\left(C_{h}, C_{l}\right)= \begin{cases}C_{h}, & \text { if }\left(C_{l} \cap C_{h}=\emptyset\right) \text { or }\left(C_{l} \supseteq C_{h}\right) \\ C_{l}, & \text { if } C_{l} \subseteq C_{h} \\ C_{h} \backslash\left(C_{h} \cap C_{l}\right), & \text { otherwise }\end{cases}
$$

Now, given the bound constraints for foldovers $\left(C_{F}\right)$, smooth changes $\left(C_{S}\right)$ and looping behaviour $\left(C_{L}\right)$ we define the final bounds as $F\left(C_{L}, F\left(C_{F}, C_{S}\right)\right)$ that give priority first to looping, then foldovers and finally smooth changes (looping is foldover-free).

\subsection{Non-linear optimisation}

We use the Levenberg-Marquardt algorithm [15], as it can efficiently calculate local minima subject to the previously described bound constraints. The squared differences that are minimised are the horizontal and vertical cell distortion energy integrals (eq. 2). The unknowns vector is the aggregation of all horizontal and vertical lines except the four that lie on the boundary. The starting point is the calculated solution from the nearest frame, as we want solutions to be as local as possible.

\section{RENDERING}

When rendering, given the original and deformed grid lines, there are two options for applying the adjusted parameterisation: altering the geometry or altering the texture coordinates. Let $F_{S}(\mathbf{s})=\mathbf{s}^{\prime}$ and $F_{t}(\mathbf{t})=\mathbf{t}^{\prime}$ be the piecewise-linear functions that remap the original to the optimised grid. As both are strictly monotonic, they can be easily inverted $\left(F_{s}^{-1}, F_{t}^{-1}\right)$.

To alter the geometry, we use the texture coordinates $T(s, t)$ with the remapped deformed geometry $D\left(F_{S}(s), F_{t}(t)\right)$. Similarly, to alter the texture coordinates, we use the deformed geometry $D(s, t)$ with the inversely remapped texture coordinates $T\left(F_{s}^{-1}(s), F_{t}^{-1}(t)\right)$.

Such a remapping is very efficient, but there are trade-offs to using any of the two methods above. If the geometry is altered, the ROI needs to be densely discretised (or dynamically tessellated with newer GPUs) and $D(s, t)$ needs to be known for the entire ROI. If the texture coordinates are altered, $T(s, t)$ needs to be known for the entire ROI.

\section{HYBRID CPU-GPU OPTIMISATION}

We use the ALGLIB library [1] for the bound-constrained LevenbergMarquardt optimiser that it provides, while we provide an objective function that calculates the squared errors and the Jacobian on the GPU.

The $O_{s}^{\prime}$ and $O_{t}^{\prime}$ functions are precalculated and stored in a texture during the pre-processing stage after the selection of the ROI. At the start of the optimisation, the $D_{s}^{\prime}$ and $D_{t}^{\prime}$ functions are calculated and stored in a texture as well.

The distortion energy integral in the objective function is calculated using a DirectCompute shader. $(M-1) \times(N-1)$ thread groups are dispatched (one per cell) and in each group a $K \times L$ grid of threads is executed ( $\max$ size $32 \times 32$ ). The thread grid for a cell is used to calculate the integral: the cell is uniformly split to $K \times L$ sub-cells and the two integrals $E_{S_{i} j}$ and $E_{t_{i} j}$ are evaluated using the midpoint method. The sub-cell results are summed using a GPU reduction operator [24] and are read back in the CPU. All required calcu- 
lations are two texture fetches, adds and multiplies, so the shader evaluation is very efficient.

The Jacobian is calculated numerically in the same shader using finite differences. As the warp is piecewise-linear, the energy for each cell is affected only by the adjacent to the cell grid lines. More specifically, $E_{s_{i j}}$ is only affected by changes of $s_{j}$ and $s_{j+1}$ and similarly $E_{t_{i j}}$ is only affected by changes of $t_{i}$ and $t_{i+1}$. As a result, the cost of calculating the Jacobian is only almost four times more than a single error calculation.

In a cell $\left(\left[s_{0}, s_{1}\right],\left[t_{0}, t_{1}\right]\right)$, given a very small offset $h$ the subcell error is additionally calculated four more times for four modified cells: $\left(\left[s_{0}+h, s_{1}\right],\left[t_{0}, t_{1}\right]\right),\left(\left[s_{0}, s_{1}-h\right],\left[t_{0}, t_{1}\right]\right),\left(\left[s_{0}, s_{1}\right],\left[t_{0}+h, t_{1}\right]\right)$, $\left(\left[s_{0}, s_{1}\right],\left[t_{0}, t_{1}-h\right]\right)$.

The integral calculation can efficiently handle the additional cell coordinates, as $f$ and $g$ (and their derivatives), being linear in nature, can be analytically adjusted for the new interval.

\section{NON-AXIS-ALIGNED GRID WARPING}

The problem can be slightly altered to allow non-axis-aligned grid warping. Given the per-cell bilinear warping functions $S_{b}(s, t)$, $T_{b}(s, t)$ and defining $H_{s}(s, t)=D\left(S_{b}(s, t), T_{b}(s, t)\right)$, equations 3,4 become:

$$
\begin{aligned}
& F_{s}(s, t)=\left(\left\|H_{s}^{\prime}(s, t)\right\|_{2}-\left\|O_{s}^{\prime}(s, t)\right\|_{2}\right)^{2} \\
& F_{t}(s, t)=\left(\left\|H_{t}^{\prime}(s, t)\right\|_{2}-\left\|O_{t}^{\prime}(s, t)\right\|_{2}\right)^{2}
\end{aligned}
$$

where

$$
\begin{aligned}
& H_{s}^{\prime}(s, t)=S_{b_{s}}^{\prime} D_{s}^{\prime}\left(S_{b}(s, t), T_{b}(s, t)\right)+T_{b_{s}}^{\prime} D_{t}^{\prime}\left(S_{b}(s, t), T_{b}(s, t)\right) \\
& H_{t}^{\prime}(s, t)=S_{b_{t}}^{\prime} D_{s}^{\prime}\left(S_{b}(s, t), T_{b}(s, t)\right)+T_{b_{t}}^{\prime} D_{t}^{\prime}\left(S_{b}(s, t), T_{b}(s, t)\right)
\end{aligned}
$$

It is straightforward to modify the boundary constraints to take into account the increased number of neighbours per point (four instead of two). The efficiency of the error calculations is reduced, as instead of directly sampling the partial derivative lengths (eq. 3, 4) we need to sample the partial derivative vectors, scale them and calculate their norms (eq. 7, 8). The Jacobian calculation process is identical, but in this case each per-axis cell energy is affected by all four adjacent points. An example of the non-axis-aligned version can be seen in figure 3 .

\section{RESULTS}

We validated the algorithm on a real dataset (face animation data from motion capture: 615 (sequence "face90") and 1373 (sequence "face49") frames, 8,820 vertices and 17,216 triangles for data in figures 1, 2, 4 and 8) as well as procedurally defined geometry. The procedural examples (figures 3, 5 and 9) used 100 frames, 16,384 vertices and 32, 258 triangles. We authored distortion control maps for all cases. In the face animation data the results are more subtle, as the captured data did not exhibit any extreme but localised deformation. For all the distortion control map features we used (bounding) cirles, as the exact shape in these maps does not have a significant effect in the algorithm results, as it mostly affects the grid generation process.

The optimisation process is non-linear and the time required for the calculation of a solution for a given frame depends on the number

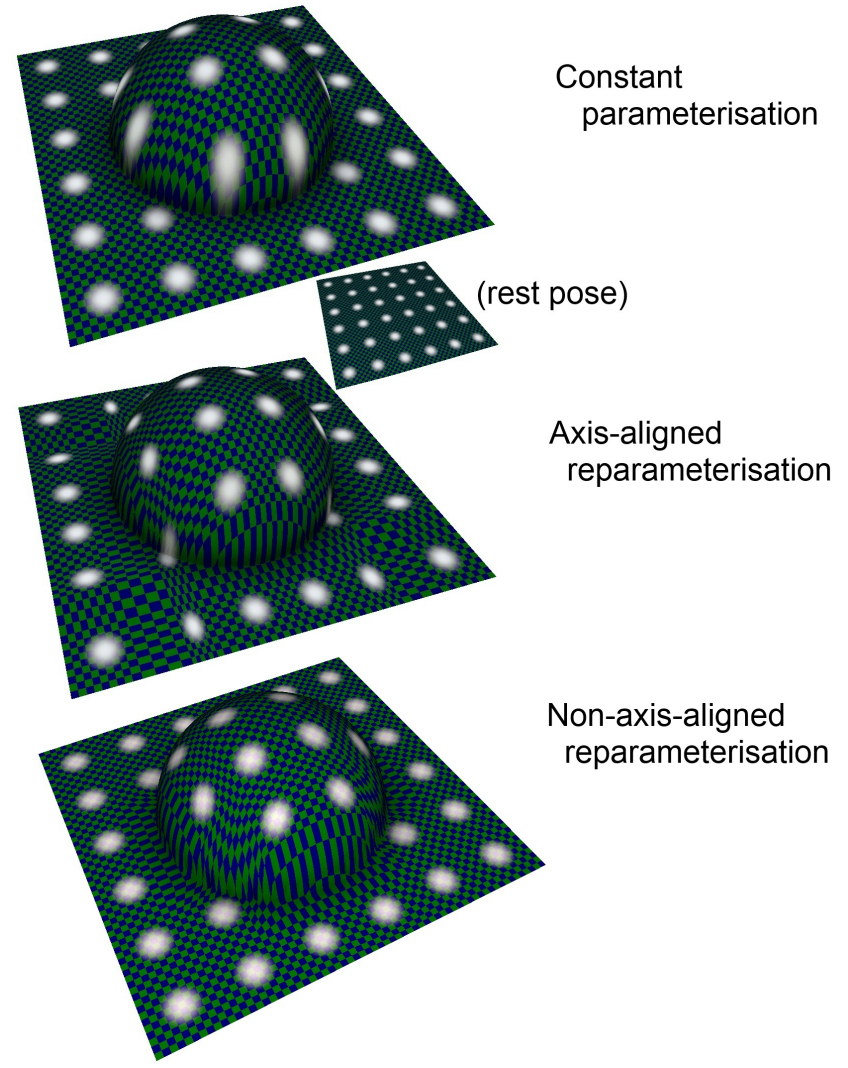

Figure 5: The original mesh is shown minimised in the top-middle. The deformation introduces creases on the mesh (top, middle and bottom). Normal texture mapping results in significant stretch of detail on the bump (top). Our axis-aligned solution reduces the stretch on features on the bump, but introduces other artifacts (middle). The optimizer converges to a suboptimal solution where some features are located on the crease. Also, due to the axis-aligned nature of the process, varying deformation across a strip results in distortions of features in undeformed areas. This can be observed here as compression of features outside the bump as a side-effect of stretch reduction of features on the bump. The non-axis-aligned version of our algorithm correctly preserves the features (bottom).

of unknowns, the complexity of deformation as well as optimisation parameters. Optimisation and remapping times are be shown in table 1 , and were measured using a $2.66 \mathrm{GHz}$ Xeon CPU with 24 GB RAM and an NVIDIA GeForce GTX 580 with 4GB VRAM. The ALGLIB optimiser settings used are for highest quality: all tolerances are set to 0 and the maximum iterations are set to 100 .

Even though the optimisation algorithm can be used in real-time on a similar hardware configuration, the results can also be precomputed and efficiently stored for use on less powerful hardware. The storage cost needed for a single ROI is $(M+N-4)$ floats, multiplied by the number of frames. So, 100 frames of animation for a moderately partitioned region (e.g. $10 \times 10$ ) would require about 6 KB. Similarly, storage for a non-axis-aligned grid would be $25 \mathrm{~KB}$. As such, the small storage costs makes the technique ideal for use in low GPU bandwidth hardware.

\section{DISCUSSION}




\begin{tabular}{cccccc} 
Example & Solver(msec) & ObjFunc (msec) & Evals $(\mathrm{Jac})$ & Unknowns & Render(ms) \\
\hline aa-face90-umouth & 9.85 & 8.49 & $18(4)$ & 14 & $1(1.35)$ \\
aa-face90-lmouth & 10.17 & 8.93 & $16(3)$ & 14 & $1(1.5)$ \\
aa-face49-leye & 22.26 & 20.7 & $23(6)$ & 12 & $1(1.3)$ \\
aa-face49-reye & 26.34 & 24.39 & $28(8)$ & 12 & $1(1.3)$ \\
aa-face49-all & 68.62 & 62.51 & $85(21)$ & $(14,14,12,12)$ & $1.1(1.8)$ \\
aa-saddle & 26.97 & 22.3 & $25(8)$ & 24 & $2.8(5)$ \\
nonaa-face90-umouth & 22.66 & 13.96 & $19(6)$ & 48 & $1(1.35)$ \\
nonaa-face90-lmouth & 54.84 & 23.16 & $20(7)$ & 80 & $1(1.5)$ \\
nonaa-saddle & 741.8 & 108.4 & $30(13)$ & 288 & $2.8(5)$ \\
aa-worm & 8.87 & 13.41 & $17(3)$ & 38 & $3.2(5.2)$ \\
nonaa-worm & 3078.15 & 425.170 & $27(15)$ & 192 & $3.2(5.2)$ \\
\hline
\end{tabular}

Table 1: Per-frame timings for various examples. The example "aa-face49-all" uses combined times for all four ROIs of the face49 animation sequence. Each distortion control map is associated with a number of unknowns for the non-linear minimizer, which is $M+N-4$ for the axis-aligned version (aa-) and $(M-2) \times(N-2) \times 2$ for the non-axis-aligned version (nonaa-). The solver times correspond to the times required for the whole optimisation (CPU-GPU). The ObjFunc times correspond to the times required for all calculations of the objective function in the GPU, with and without Jacobian calculation, including the transfers to the CPU. The Evals column shows the average number of objective function evaluations per solved frame, while the number in the parentheses shows the average number or required Jacobian evaluations per solved frame. The rendering times correspond to close-up views of highly tessellated geometry using the modified and original parameterisations (numbers inside and outside parentheses respectively). Rendering times for the modified parameterisation include rendering the parameterisation to a texture and sampling it from the normal shader used for the mesh. It can be seen that even though the objective function calculation times scale well with the number of the unknowns, the rest of the optimisation does not (especially for the non-axis-aligned variant) so for larger problem sizes the performance deteriorates quickly. These examples have not been optimised for performance, as all tolerances are zero.

In this section we discuss various issues and capabilities of the proposed algorithm.

\subsection{Grid lines and filtering}

To avoid filtering artefacts, when calculating the initial non-uniform grid, we must ensure that when separating a low-rigidity from a high-rigidity cell, the separating line must move a few pixels towards the low-rigidity one, as otherwise texture filtering can cause stretching of rigid detail. An additional consequence is that using hardware trilinear filtering can also cause artifacts, as sampling from low resolution mipmaps can result in rigid detail bleeding in a non-rigid (and potentially highly deforming) neighbouring area.

\subsection{Edge discontinuities}

Remapping of axis-aligned lines results in seams on the edges of the selected ROI, as the parameterisation there will be discontinuous. This can easily be avoided by doing the following: a) ensure the cells of the rectilinear grid that are adjacent to the boundary contain non-rigid data and b) at the boundary cells, linearly blend the original and optimised parameterisation so that when approaching a vertical edge the $\mathbf{s}^{\prime}$ solution blends to $\mathbf{s}$ and when approaching a horizontal edge the $\mathbf{t}^{\prime}$ solution blends to $\mathbf{t}$.

\subsection{ROIs and chart boundaries}

If a selected ROI contains a texture space region which is unused, the resulting distortion control weight and partial derivative length textures will contain the texture initialisation values, which should be zero. In this case, the calculated energy values (eq. 3 and 4 ) in those areas are 0 and do not affect the rest of the process.

\subsection{Failure cases}

There are cases where the algorithm fails, such as when distortion on the deformed surface varies signicantly across a horizontal or vertical strip (figure 6). Additionally, high-frequency deformation (e.g. by introducing creases on previously smooth areas) will result in slower convergence or lower quality results, especially for the axis-aligned version of the algorithm (figure 5).

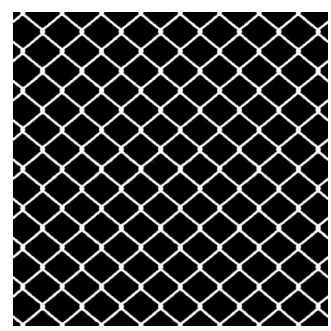

Figure 6: An example of a distortion control map that cannot be used successfully with the algorithm.

\section{CONCLUSION AND FUTURE WORK}

In this paper we presented a technique to reparameterise regions in texture space, so that important rigid features mapped on these regions are preserved when the surface deforms. The proposed algorithm achieves good results, requires minimal user interaction and exhibits fast computation and runtime evaluation as well as very low storage requirements.

The technique can be applied to reduce elastic distortions on a variety of scenarios where highly detailed rigid features are represented on a map, abstracted from the underlying low-complexity deforming geometry they are mapped on. In modeling packages, artists can preview animations with detail mapped in a content-aware way, without manually altering the model geometry to achieve that. Realtime animated rigid detail on deformable objects also becomes a possibility, whereas previously it would require a significant amount of work from artists. Precomputed remappings for canned animations can enhance visual quality by reducing the rigid detail distortion on deformable surfaces in low-power hardware. In general, 
control cages/sparse meshes with static or dynamic detail are ideal candidates for use with this method, as the geometry remains unchanged and as a result it can be shared with more detail maps, requiring only precalculated parameterisation corrections for ROIs for each detail map.

The piecewise-linear warp is $C 0$-continuous on the grid edges, so we would like to modify the algorithm so that the remapping is at least $C 1$-continuous in the domain of the ROI, resulting in a higherquality reparameterisation.

Additionally, the requirement for a rectilinear grid is too restrictive, so we would like to generalize the non-uniform-grid formulation to handle arbitrary mesh connectivity: in that case, the ROI can have an arbitrary shape and the warping mesh can be carefully constructed to minimize optimisation time.

We would also like to extend the algorithm to perform contentaware warp of the volumetric space (thick shell over the surface), so that detail of any complexity can be preserved under deformation.

\section{REFERENCES}

[1] ALGLIB. www.alglib.net.

[2] M. Ben-Chen, O. Weber, and C. Gotsman. Variational harmonic maps for space deformation. In ACM Transactions on Graphics (TOG), volume 28, page 34. ACM, 2009.

[3] M. Botsch and O. Sorkine. On linear variational surface deformation methods. Visualization and Computer Graphics, IEEE Transactions on, 14(1):213-230, 2008.

[4] B. Burley and D. Lacewell. Ptex: Per-face texture mapping for production rendering. In Computer Graphics Forum, volume 27, pages 1155-1164. Wiley Online Library, 2008.

[5] M. Floater and K. Hormann. Surface parameterization: a tutorial and survey. Advances in multiresolution for geometric modelling, pages 157-186, 2005.

[6] R. Gal, O. Sorkine, and D. Cohen-Or. Feature-aware texturing. In Proceedings of Eurographics Symposium on Rendering, pages 297-303, 2006.

[7] P. Heckbert. Survey of texture mapping. Computer Graphics and Applications, IEEE, 6(11):56-67, 1986.

[8] K. Hormann, B. Lévy, A. Sheffer, et al. Mesh parameterization: Theory and practice. SIGGRAPH Course Notes, 2007.

[9] A. Jacobson, I. Baran, J. Popovic, and O. Sorkine. Bounded biharmonic weights for real-time deformation. SIGGRAPH'11: ACM SIGGRAPH 2011 Papers, 2011.

[10] P. Joshi, M. Meyer, T. DeRose, B. Green, and T. Sanocki. Harmonic coordinates for character articulation. $A C M$ Transactions on Graphics (TOG), 26(3):71, 2007.

[11] T. Ju, S. Schaefer, and J. Warren. Mean value coordinates for closed triangular meshes. In ACM Transactions on Graphics (TOG), volume 24, pages 561-566. ACM, 2005.

[12] V. Kraevoy, A. Sheffer, A. Shamir, and D. Cohen-Or. Non-homogeneous resizing of complex models. In $A C M$ Transactions on Graphics (TOG), volume 27, page 111. ACM, 2008.

[13] Y. Lipman, D. Levin, and D. Cohen-Or. Green coordinates. In ACM Transactions on Graphics (TOG), volume 27, page 78. ACM, 2008.

[14] J. Maillot, H. Yahia, and A. Verroust. Interactive texture mapping. In Proceedings of the 20th annual conference on Computer graphics and interactive techniques, pages 27-34.
ACM, 1993.

[15] J. More. The levenberg-marquardt algorithm: implementation and theory. Numerical analysis, pages 105-116, 1978.

[16] D. Panozzo, O. Weber, and O. Sorkine. Robust image retargeting via axis-aligned deformation. In Computer Graphics Forum, volume 31, pages 229-236. Wiley Online Library, 2012.

[17] T. Popa, D. Julius, and A. Sheffer. Material-aware mesh deformations. In Shape Modeling and Applications, 2006. SMI 2006. IEEE International Conference on, pages 22-22. IEEE, 2006.

[18] P. Sander, S. Gortler, J. Snyder, and H. Hoppe. Signal-specialized parametrization. In Proceedings of the 13th Eurographics workshop on Rendering, pages 87-98. Eurographics Association, 2002.

[19] A. Sheffer and E. De Sturler. Smoothing an overlay grid to minimize linear distortion in texture mapping. $A C M$ Transactions on Graphics (TOG), 21(4):874-890, 2002.

[20] A. Sheffer, E. Praun, and K. Rose. Mesh parameterization

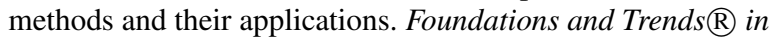
Computer Graphics and Vision, 2(2):105-171, 2006.

[21] O. Sorkine. Laplacian mesh processing. In Eurographics State-of-the-Art Report, pages 53-70, 2005.

[22] W. Xu and K. Zhou. Gradient domain mesh deformation - a survey. Journal of computer science and technology, 24(1):6-18, 2009.

[23] X. Yang, R. Southern, and J. Zhang. Fast simulation of skin sliding. Computer Animation and Virtual Worlds, 20(2-3):333-342, 2009.

[24] E. Young. Directcompute optimisations and best practices. In GPU Technology Conference, 2010.

\section{APPENDIX}

\section{A. DERIVATION OF ENERGY FOR AXIS- ALIGNED DEFORMATION}

We want identical lengths for a small segment $\left(s_{0}, s_{1}\right)$, where the geometry function can be considered as piecewise-linear.

$$
\left\|D\left(s_{1}^{\prime}\right)-D\left(s_{0}^{\prime}\right)\right\|=\left\|O\left(s_{1}\right)-O\left(s_{0}\right)\right\|
$$

Because $s^{\prime}=f(s)=c s+d$, the above can be further rewritten as:

$$
\left\|D\left(f\left(s_{1}\right)\right)-D\left(f\left(s_{0}\right)\right)\right\|=\left\|O\left(s_{1}\right)-O\left(s_{0}\right)\right\|
$$

The function $f(s)$ can be also written as follows:

$$
f(s)=s_{0}^{\prime}+\frac{s-s_{0}}{s_{1}-s_{0}}\left(s_{1}^{\prime}-s_{0}^{\prime}\right)
$$

and its derivative in this case is:

$$
f^{\prime}(s)=\frac{s_{1}^{\prime}-s_{0}^{\prime}}{s_{1}-s_{0}}
$$

Divide by $s_{1}-s_{0}$ to be able to convert it to derivatives: 


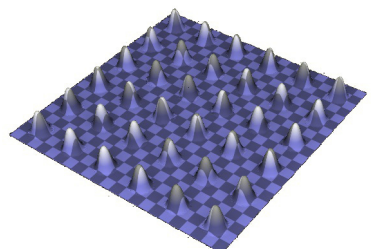

(a) Rest pose

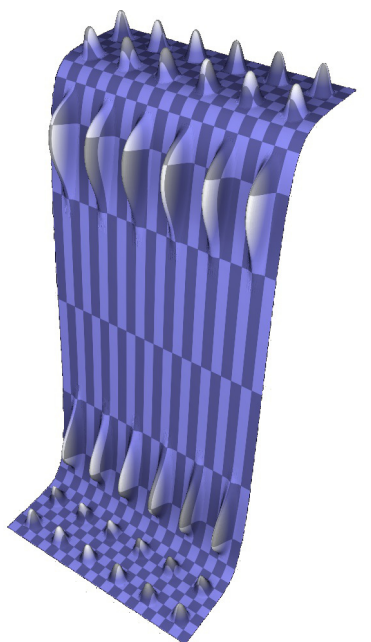

(b) Deformed, using original parameterisation
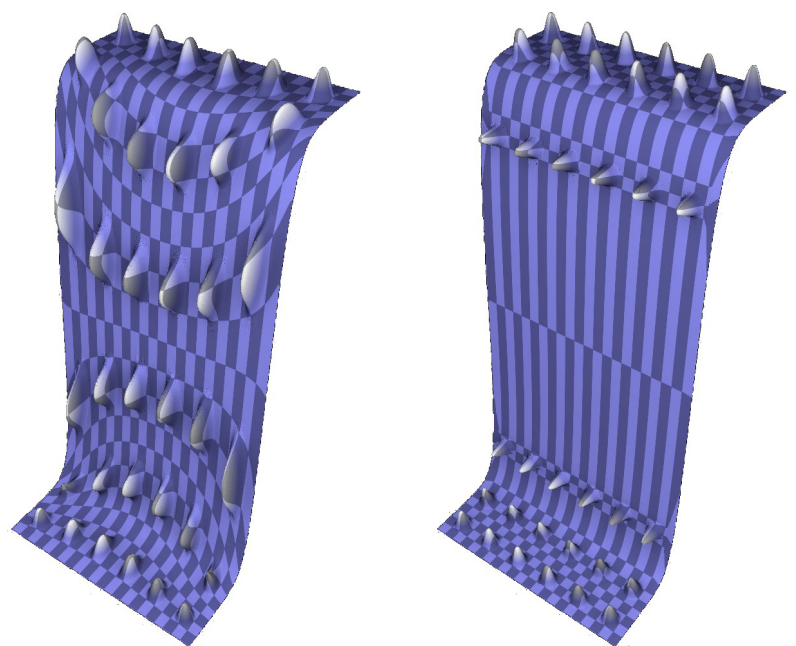

(c) Deformed, reparameterised using (d) Deformed, using content-aware CGAL's mean value coordinates reparameterisation

Figure 7: Fixed plane deformation with mapped displacements. The constant parameterisation ( 7b) stretches the features. Reparameterising the mesh using an off-the-shelf method ( 7c) does not preserve the features. Reparameterising the mesh using our method ( $7 \mathrm{~d}$ )preserves the features and spreads the error in non-feature regions.

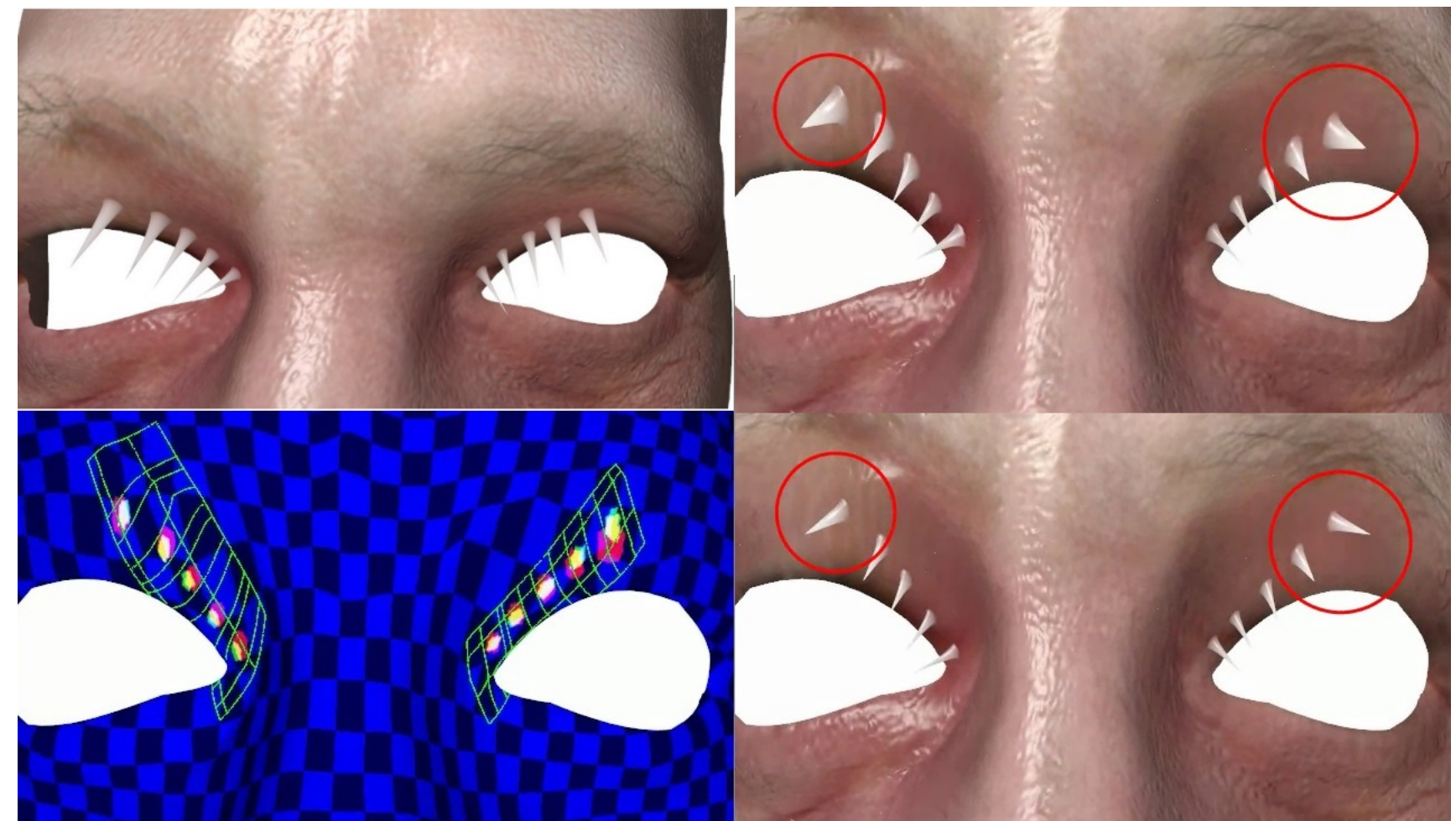

Figure 8: Mapped features near the eyes. The rest pose is shown at the top-left. The features stretch under deformation (top-right). Our algorithm calculates a reparameterisation that reduces distortions near the features (bottom-right). The remapped ROI rectangle and the features in the original and remapped parameterisation (red and green respectively) are shown at the bottom-left. 


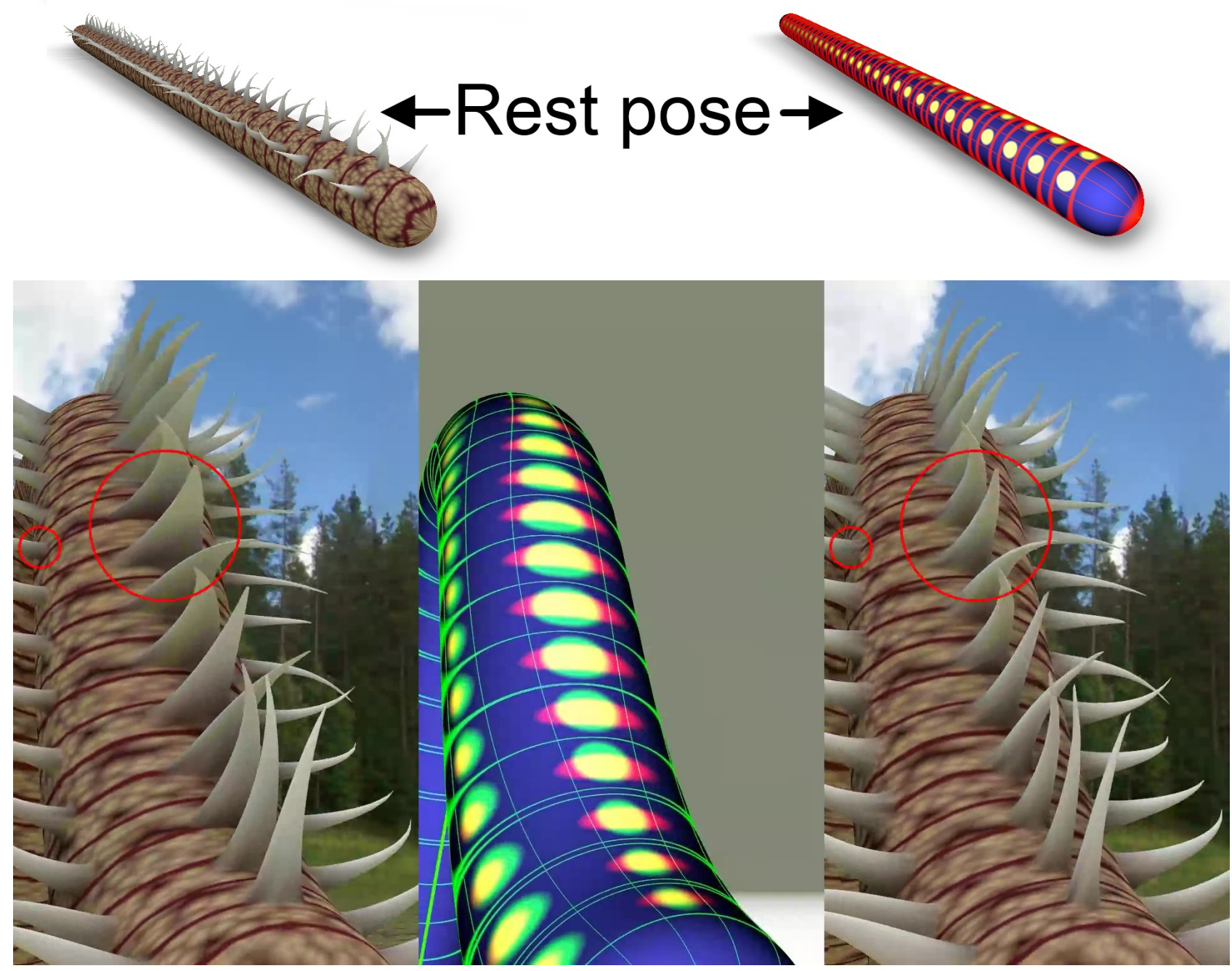

Figure 9: Worm example. Rest pose is shown on top, with the distortion control map. On the left, the deformed pose compresses the features near the center of the worm. On the right, the parameterisation is warped so that the parameterisation distortion near the features is spread to non-feature areas. In the middle, the areas of interest in the original and content-aware parameterisation are shown in red and green respectively.

$$
\frac{\left\|D\left(f\left(s_{1}\right)\right)-D\left(f\left(s_{0}\right)\right)\right\|}{s_{1}-s_{0}}=\frac{\left\|O\left(s_{1}\right)-O\left(s_{0}\right)\right\|}{s_{1}-s_{0}}
$$

Rewriting $H(x)=D(f(x))$ leads to:

$$
\begin{aligned}
\frac{\left\|H\left(s_{1}\right)-H\left(s_{0}\right)\right\|}{s_{1}-s_{0}} & =\frac{\left\|O\left(s_{1}\right)-O\left(s_{0}\right)\right\|}{s_{1}-s_{0}} \\
\left\|H^{\prime}(s)\right\| & =\left\|O^{\prime}(s)\right\| \\
\left|f^{\prime}(x)\right|\left\|D^{\prime}(f(x))\right\| & =\left\|O^{\prime}(s)\right\| \\
\left|f^{\prime}(x)\right|\left\|D^{\prime}(f(x))\right\|-\left\|O^{\prime}(s)\right\| & =0
\end{aligned} \quad \Rightarrow
$$

and this leads to the equations 3 and 4 . 